Purdue University

Purdue e-Pubs

CTRC Research Publications

Cooling Technologies Research Center

2005

\title{
Influence of Bulk Fluid Velocity on the Efficiency of Electrohydrodynamic Pumping
}

V. Singhal

S V. Garimella

Purdue University, sureshg@purdue.edu

Follow this and additional works at: http://docs.lib.purdue.edu/coolingpubs

Singhal, V. and Garimella, S V., "Influence of Bulk Fluid Velocity on the Efficiency of Electrohydrodynamic Pumping" (2005). CTRC Research Publications. Paper 289.

http://dx.doi.org/10.1115/1.1899173

This document has been made available through Purdue e-Pubs, a service of the Purdue University Libraries. Please contact epubs@purdue.edu for additional information. 


\author{
Vishal Singhal \\ Suresh V. Garimella \\ e-mail: sureshg@ecn.purdue.edu \\ School of Mechanical Engineering, \\ Purdue University, \\ 585 Purdue Mall, \\ West Lafayette, IN 47907-2088
}

\section{Influence of Bulk Fluid Velocity on the Efficiency of Electrohydrodynamic Pumping}

\begin{abstract}
The efficiency of conversion of electrical power into fluidic power in an electrohydrodynamic (EHD) pump depends on the bulk fluid velocity. An analytical formulation is developed for calculation of the efficiency of an EHD pump, with and without the presence of a superimposed flow due to an externally imposed pressure gradient. This formulation is implemented into a numerical model, which is used to investigate the effect of bulk fluid velocity on the efficiency of the EHD action. In particular, the net flow due to the combined action of EHD and a positive or negative external pressure gradient is computed. Both ion-drag pumps and induction EHD pumps are considered. Pumps based on the ion-drag principle that are studied include a one-dimensional pump, a twodimensional pump driven by a stationary potential gradient, and another driven by a traveling potential wave. Two-dimensional repulsion-type and attraction-type induction pumping caused by a gradual variation in the electrical conductivity of the fluid is also investigated. The efficiency of EHD pumps exhibited a strong dependence on bulk fluid velocity: for the two-dimensional steady ion-drag pump, for example, the efficiency increased from less than $2 \%$ to $22 \%$ under the influence of an external pressure gradient. The corresponding increase in efficiency for a two-dimensional repulsion-type EHD pump was from $0.26 \%$ to $24.5 \%$. [DOI: 10.1115/1.1899173]
\end{abstract}

\section{Introduction}

Electrohydrodynamics (EHD) as a means of pumping fluids has been under investigation for several decades [1-5]. However, the low efficiency of conversion of electrical power into fluid power has limited the implementation of EHD pumps in practical applications. Recent developments in microfluidics have led to a renewed interest in EHD pumps mainly because of their potential for miniaturization, absence of moving parts, and the resulting high reliability. Miniature ion-drag [6,7], induction EHD [8,9] and electro-osmotic $[10,11]$ pumps have been investigated. However, the efficiency of these devices, seldom greater than $5 \%$ and often less than $1 \%$, continues to be a critical issue of concern.

The efficiency of an EHD pump strongly depends on the bulk fluid velocity. Specifically, if an ion-drag pump is operated under an external pressure gradient, causing flow in the same direction as the EHD pump, the efficiency of conversion of electrical power to fluid power is higher than if the external pressure gradient were not present [12]. Conversely, an external pressure gradient in the opposite direction causes a decrease in EHD pumping efficiency. This effect can be used to advantage. For instance, EHD pumping could be used as a booster for existing flow inside pipes or channels. The main pumping action would be carried out by an external pump, while the EHD action helps to increase the fluid velocity. Control of local heat transfer in specific regions or tubes in a heat exchanger, for example, by this means could be of great advantage in specific applications.

Several studies have considered the efficiency of EHD pumps [13-15]. Most, however, have focused on the effect of the electrical properties of the fluid on the efficiency of conversion of electrical power to fluidic power. The role of bulk fluid velocity in increasing the efficiency of EHD pumps has not received much attention. The study of Bondar and Bastien [12] appears to be the only one that has identified the potential increase in the efficiency of EHD action due to increased bulk fluid velocity.

Contributed by the Fluids Engineering Division for publication in the JouRnal of Fluids EngineERING. Manuscript received by the Fluids Engineering Division April 20, 2004; revised manuscript received March 5, 2005. Associate Editor: Akira Goto.
A transient, three-dimensional model of electrohydrodynamics, capable of solving coupled charge transport and Navier-Stokes equations, was recently developed $[16,17]$. This model is used here to study the effect of bulk fluid velocity on the efficiency of conversion of electrical power into fluidic power in an EHD pumping device. One-dimensional (1D) and two-dimensional iondrag pumps actuated using a stationary potential gradient as well as a traveling potential wave are studied. Attraction- and repulsion-type induction EHD pumps are also considered.

\section{Previous Studies}

A few studies in the literature have dealt specifically with the efficiency of EHD pumps. Crowley [13] studied the efficiency of EHD induction pumps which use the electrical conductivity jump at the interface between different fluids for inducing charges. This study was confined to the attraction mode, where fluid motion is in the same direction as the traveling potential wave. This would occur when the electric field strength is higher in the fluid with the smaller electrical conductivity. Flow between two parallel plates was studied. Layers of two nonmixing fluids with different electrical properties were present between the plates. A traveling potential wave was applied to the plate which was in contact with the fluid of lower electrical conductivity. The other plate was grounded. An analytical expression was derived for the efficiency of the pump, as a ratio of the product of average shear stress at the interface and velocity of the interface to the time-averaged electrical power input. The effect on pump efficiency of various parameters was analyzed using the expression derived. The efficiency was indicated to be high under the following conditions: the charge relaxation time in the less-conducting fluid is smaller than the time period of the potential wave; the more conducting fluid is highly conductive; and the less-conducting fluid layer is thin, with thickness much smaller than the wavelength of the potential wave.

Bondar and Bastien [12] presented experimental results on the effect of bulk fluid velocity on the efficiency of EHD. Ions were generated by corona discharge from a moving pointed electrode attached to a steel rod. The force due to electrical interaction 
between the charges and the rod caused a variation in the acceleration of the steel rod. The resultant change in velocity was measured using an opto-electronic detector. This experiment was performed without and with a pressurized air stream, with the efficiency reaching $2.6 \%$ and $7.5 \%$ under the former and latter conditions, respectively. This compared to an efficiency of less than $1 \%$ without the pressurized air and moving electrode. The efficiency increase was also found to be independent of the electrical power input to the corona discharge. They also presented an integral equation for the efficiency of a steady (constant voltage drop and charge source) EHD pump. Assuming negligible viscous losses in the ionization region, it was shown that the efficiency of EHD can be changed solely by the bulk fluid velocity, without changes in the electrical parameters.

Crowley et al. [14] conducted a theoretical study on the effect of fluid properties on the efficiency and flow rate of a twodimensional ion-drag EHD pump. Variations in electric field due to the space charge effect were neglected, and the electric field was assumed to be uniform throughout the pump. This facilitated an analytical solution of the governing equations. The efficiency of the EHD pump was defined as $\eta=1 /(1+\alpha)$, where $\alpha$ is given by $\alpha=\mu E / v+\sigma E / q v$. These expressions were obtained from a simplified consideration that the efficiency was equal to the ratio of the electrical power input if fluid mobility and conductivity were zero, to the actual electrical power input, i.e., $\eta$ $=q v A V_{e} /\left(q v A V_{e}+\mu q A E V_{e}+\sigma A E V_{e}\right)$. It was concluded that low electrical conductivity and low ion mobility lead to high efficiency. Limits on flow velocity in an ion-drag EHD pump due to several different factors were also identified. These factors are charge decay by charge conduction and ion mobility, friction forces for laminar and turbulent flow, and breakdown of fluid at high electric fields. Low fluid viscosity and high permittivity were also suggested to lead to high flow rates.

Seyed-Yagoobi et al. [15] presented a theoretical model of steady 1D EHD pumping. Current due to conduction, mobility, and convection of charges was accounted for in the governing equations for EHD. For a 1D flow the EHD equations can be solved without recourse to Navier-Stokes equations. This is because there are no pressure gradients or viscous losses in a 1D flow and hence the flow velocity is the same everywhere. The charge transport equation was solved numerically to obtain electric field and charge density distributions which were then used to calculate efficiency. Results were presented in terms of three nondimensional numbers: Electric Reynolds number $\operatorname{Re}_{\mathrm{E} \ell}=\varepsilon v / \sigma L$, Electric slip number $E s l=\mu V_{e} / v L$, and Electric source number $E s=q_{e} L^{2} / \varepsilon V_{e} ; \mathrm{Re}_{\mathrm{E} \ell}$ is the ratio of free-charge relaxation time of the fluid $(\varepsilon / \sigma)$ to the time which characterizes system dynamics $(L / v), E s l$ represents the relative motion of charges compared to the bulk fluid velocity, and Es indicates the influence of space charge on the electric field. The Electric Reynolds number $\operatorname{Re}_{\mathrm{E} \ell}$ is also indicative of the efficiency of energy conversion. For a steady 1D EHD pump with an applied voltage difference across a domain and a constant known charge density upstream of the domain, the efficiency of the pump was given by $\eta=0.5\left(E_{c}^{* 2}-E_{e}^{* 2}\right) /\left(\operatorname{Re}_{\mathrm{E} \ell} E_{c}^{*}\right.$ $\left.+E s \rho_{c}^{*}-E s E s l \rho_{c}^{*} E_{c}^{*}\right)$, where $E_{c}^{*}$ and $E_{e}^{*}$ are the nondimensional electric fields downstream (at collector) and upstream (at emitter) of the domain and $\rho_{c}^{*}$ is the nondimensional charge density downstream of the EHD pump. The quantities $E_{c}^{*}, E_{e}^{*}$, and $\rho_{c}^{*}$ were obtained from numerical analysis. For given values of $E s$ and $E s l$, efficiency was shown to increase with $\operatorname{Re}_{\mathrm{E} \ell}$. The efficiency was higher for low values of Esl, which corresponds to low mobility, small voltage difference, or large fluid velocity. Es was shown to be important only at low values of $\operatorname{Re}_{\mathrm{E} \ell}$. It was thus concluded that low mobility, low conductivity, and high permittivity all lead to higher efficiency.

Most of the related studies in the literature have focused on the effect of electrical properties of the fluid on EHD efficiency. To the authors' knowledge, Bondar and Bastien [12] reported the only study which identified bulk fluid velocity as a significant parameter in determining the efficiency of EHD. Moreover, the theoretical and numerical analyses in past studies have been limited to very simplified systems, with simplifying approximations frequently made in the governing equations. This was necessitated due to the inability to solve coupled charge transport and NavierStokes equations in these studies, which is required to calculate the efficiency of an EHD system.

A theoretical model for EHD pumping is developed below, following which the numerical analysis approach is described, including model validation results. The variation of the efficiency of a number of EHD pump configurations is then explored as a function of bulk fluid velocity.

\section{Theoretical Analysis}

A methodology for the calculation of efficiency of EHD pumping is developed. Alternative definitions for the efficiency of an EHD pump in the presence of an externally imposed bulk fluid velocity are presented. General integral equations are derived for both definitions of efficiency, and then simplified for the particular systems considered here.

Governing Equations. Magnetic induction due to moving charges is assumed to be negligible in the following discussion, which means the electric field $\mathbf{E}$ is irrotational. Gauss's law can be written in terms of the electric potential $\Phi(V)$ as

$$
q=-\nabla \cdot(\varepsilon \nabla \Phi)
$$

Conservation of charge $q$, in the absence of any charges due to species reaction, is given by

$$
\frac{\partial q}{\partial t}+\nabla \cdot \mathbf{J}=0
$$

Here, $\mathbf{J}$, the current density vector is given by

$$
\mathbf{J}=\sigma \mathbf{E}+q \mathbf{v}+q \mu \mathbf{E}-D \boldsymbol{\nabla} q
$$

The four terms on the right hand side of Eq. (3) represent current due to conduction, convection, ionic mobility, and diffusion of charges, respectively. A detailed explanation of these terms is available in $[16,17]$. Current due to diffusion of charges is generally negligible, as the diffusion Peclet number is generally much greater than unity, as is assumed in the following analysis [15]. Otherwise, current due to charge diffusion can be significant and should not be neglected.

Combining Eqs. (2) and (3), the charge transport equation can be written as

$$
\frac{\partial q}{\partial t}+\nabla \cdot(q \mathbf{v})=\nabla \cdot(\sigma \nabla \Phi)+\nabla \cdot(q \mu \nabla \Phi)
$$

The continuity and Navier-Stokes equations which describe the fluid flow are given below. The Navier-Stokes equations are modified to include pressure generation due to Coulomb forces.

$$
\text { Continuity: } \frac{\partial \rho}{\partial t}+\boldsymbol{\nabla} \cdot \rho \mathbf{v}=0
$$

Navier-Stokes: $\frac{\partial \rho \mathbf{v}}{\partial t}+(\mathbf{v} \cdot \boldsymbol{\nabla}) \mathbf{v}=-\boldsymbol{\nabla} p+\boldsymbol{\nabla} \cdot \tau_{i j}+\rho \mathbf{f}-q \boldsymbol{\nabla} \Phi$

Body forces (other than Coulomb forces) are assumed negligible in the following analysis. The charge transport Eqs. (1) and (4) are solved along with the fluid transport Eqs. (5) and (6) via a numerical analysis to obtain the distribution of potential and charge, as well as the flow characteristics. The electrical and fluidic equations are coupled due to the presence of the charge convection term in the charge transport equation and the Coulomb force term in the Navier-Stokes equations. 
Efficiency Calculation. The efficiency of a steady-state EHD pump will be shown by the following analysis to vary with bulk fluid velocity. An expression for the efficiency of a general (steady/transient) EHD pump is then derived, and is subsequently simplified for the different pumps considered.

In a steady-state system, the electrical power input to an EHD pump is given by

$$
P_{i}=\iint_{(\mathrm{Vol})} \int_{\text {ion }} \cdot d \mathbf{F}_{\mathrm{E} \ell}
$$

Here $P_{i}$ is input (electrical) power, $\mathbf{v}_{\text {ion }}$ is velocity of the ions and $d \mathbf{F}_{\mathrm{E} \ell}$ is electrical force acting on a unit volume of the domain. The electrical force on a unit volume can be further written as $d \mathbf{F}_{\mathrm{E} \ell}$ $=\mathbf{E} d q$, where $d q$ is charge density in that unit volume. Hence, Eq. (7) can be written as

$$
P_{i}=\iiint_{(\mathrm{Vol})} \mathbf{v}_{\text {ion }} \cdot \mathbf{E} d q
$$

The mechanical power output from the EHD pump, $P_{o}$, can be written as

$$
P_{o}=\iint_{(\mathrm{Vol})} \int_{\mathbf{f}_{\mathrm{fl}}} \cdot d \mathbf{F}_{m}
$$

Here, $\mathbf{v}_{\mathrm{fl}}$ is bulk velocity of the fluid and $d \mathbf{F}_{m}$ is that component of the force which contributes to useful work.

The bulk velocity of the ions is related to the velocity of the fluid. When the ions are in equilibrium with the fluid, i.e., when the difference in bulk velocities of the ions and the fluid depends only on local electric field (as would happen when there are no external sources of ions in the domain, i.e., charges are not being created or destroyed due to induction or chemical reactions), this relation can be expressed as

$$
\mathbf{v}_{\text {ion }}=\mathbf{v}_{\mathrm{fl}}+\mathbf{v}_{\text {drift }}
$$

Here $\mathbf{v}_{\text {drift }}$ is the drift velocity of the ions and is given by $\mathbf{v}_{\text {drift }}$ $=\mu \mathbf{E}$. It should be noted that in the absence of free electrons, external sources of ions or diffusion, the equation for current density [Eq. (3)] reduces to $\mathbf{J}=q \mathbf{v}_{\mathrm{fl}}+q \mu \mathbf{E}$. Dividing this equation by charge density $q$ results in Eq. (10).

The efficiency of an EHD pump can therefore be written as

$$
\eta_{\mathrm{E} \ell}=\frac{P_{o}}{P_{i}}=\frac{\iint_{(\mathrm{Vol})} \int_{\mathrm{fl}} \cdot d \mathbf{F}_{m}}{\iiint_{(\mathrm{Vol})} \mathbf{v}_{\text {ion }} \cdot d \mathbf{F}_{\mathrm{E} \ell}}
$$

If it is assumed that $d \mathbf{F}_{m}=d \mathbf{F}_{\mathrm{E} \ell}$, which implies that there are negligible losses due to frictional forces and viscous effects, the efficiency is given by

$$
\eta_{\mathrm{E} \ell}=\frac{\iint_{(\mathrm{Vol})} \int_{(\mathrm{Vol})} \mathbf{v}_{\mathrm{fl}} \cdot d \mathbf{F}_{\mathrm{E} \ell}}{\left.\iiint_{\mathrm{fl}}+\mathbf{v}_{\mathrm{drift}}\right) \cdot d \mathbf{F}_{\mathrm{E} \ell}}
$$

This expression was first presented by Bondar and Bastien [12]. It can be further rewritten as

$$
\eta_{\mathrm{E} \ell}=\frac{v_{\mathrm{fl}, \mathrm{wa}}}{v_{\text {ion,wa }}}=\frac{1}{1+v_{\mathrm{drift}, \mathrm{wa}} / v_{\mathrm{fl}, \mathrm{wa}}}
$$

in which $v_{\text {fluid,wa }}, v_{\text {ion,wa }}$, and $v_{\text {drift,wa }}$ refer to the weighted average of fluid, ion and drift velocities with respect to the electric force density.

Equation (13) suggests that the efficiency of an EHD pump is related to bulk fluid velocity in the pump. No assumption was made in this analysis regarding the source of this fluid velocity. It may be solely due to the EHD action or may be brought about by an external pressure gradient. In fact, Eq. (13) suggests that the efficiency of an EHD pump can change radically without a change in the electrical conditions of the pump. The pump efficiency would increase with an increase in the fluid bulk velocity (if it is in the same direction as the drift velocity of the ions) and vice versa.

The instantaneous electrical power input $P_{i}$ to any EHD pump can be written as

$$
P_{i}=\iint_{(\mathrm{Vol})} \int_{\mathrm{V}} d \mathbf{V} \cdot \mathbf{I}=\int \mathbf{E} \cdot \mathbf{J} d \mathrm{Vol}
$$

The above integral would be carried out over the entire region which has a current path to any of the powered electrodes. Introducing $\mathbf{J}=\sigma \mathbf{E}+q \mathbf{v}+q \mu \mathbf{E}$ in the above equation yields

$$
P_{i}=\int \mathbf{E} \cdot(\sigma \mathbf{E}+q \mathbf{v}+q \mu \mathbf{E}) d V o l
$$

In Cartesian coordinates, Eq. (15) reduces to

$$
\begin{aligned}
P_{i}= & \iiint\left[(\sigma+q \mu)\left(E_{x}^{2}+E_{y}^{2}+E_{z}^{2}\right)+q\left(E_{x} v_{x}+E_{y} v_{y}\right.\right. \\
& \left.\left.+E_{z} v_{z}\right)\right] d x d y d z
\end{aligned}
$$

The mechanical work done by an EHD pump causes a pressure gradient in the fluid, which changes its velocity. For a fluid already in motion, the velocity would increase if the Coulomb forces are in same direction as the existing flow, and would decrease otherwise. The pressure gradient is balanced by viscous forces in the fluid and friction forces at the fluid-solid interfaces which act to retard the flow. Hence the mechanical power output $P_{o}$ of an EHD pump is given by

$$
P_{o}=\int(\mathbf{s} \cdot \mathbf{v})_{\mathrm{EHD}}-(\mathbf{s} \cdot \mathbf{v})_{\mathrm{No}} \mathrm{EHD} d S
$$

in which $\mathbf{s}$ is the stress vector. Subscript "EHD" in the equation refers to flow due to combined action of EHD forces and external pressure gradient, while subscript "No EHD" refers to flow solely due to external pressure gradient. The above integral is executed over the boundary of the domain. The retarding forces are reflected in the velocity gradients in the fluid. Pressure and viscous stresses can be written as $s_{i j}=-p_{\text {tot }} \delta_{i j}+\mu_{\text {vis }}\left(v_{i, j}+v_{j, i}\right)$, where $p_{\text {tot }}$ is total pressure drop, $\mu_{\mathrm{vis}}$ is viscosity of the fluid and $\delta_{i j}$ is the Kronecker delta vector, $\delta_{i j}=\left\{\begin{array}{c}1 \text { if } i=j \\ 0 \text { if } i \neq j\end{array}\right.$. The term $s_{i j}$ represents stress in the $j$ direction on a plane in the $i$ direction. However, flow due to an applied external pressure gradient is not reflected in the above equation, as both the pressure gradient as well as the viscous forces caused by this pressure gradient are included in the equation. The equation for $s_{i j}$ can be modified as follows to account for flow due to an applied external pressure gradient $s_{i j}=$ $-p \delta_{i j}+\mu_{\mathrm{vis}}\left(v_{i, j}+v_{j, i}\right)$, where $p=p_{\text {tot }}-p_{\text {app }}$ is net pressure gradient generated by the EHD pump ( $p_{\text {app }}$ is applied external pressure gradient).

The output fluid power can therefore be written as 
Table 1 Description of different EHD pumps considered in the present study.

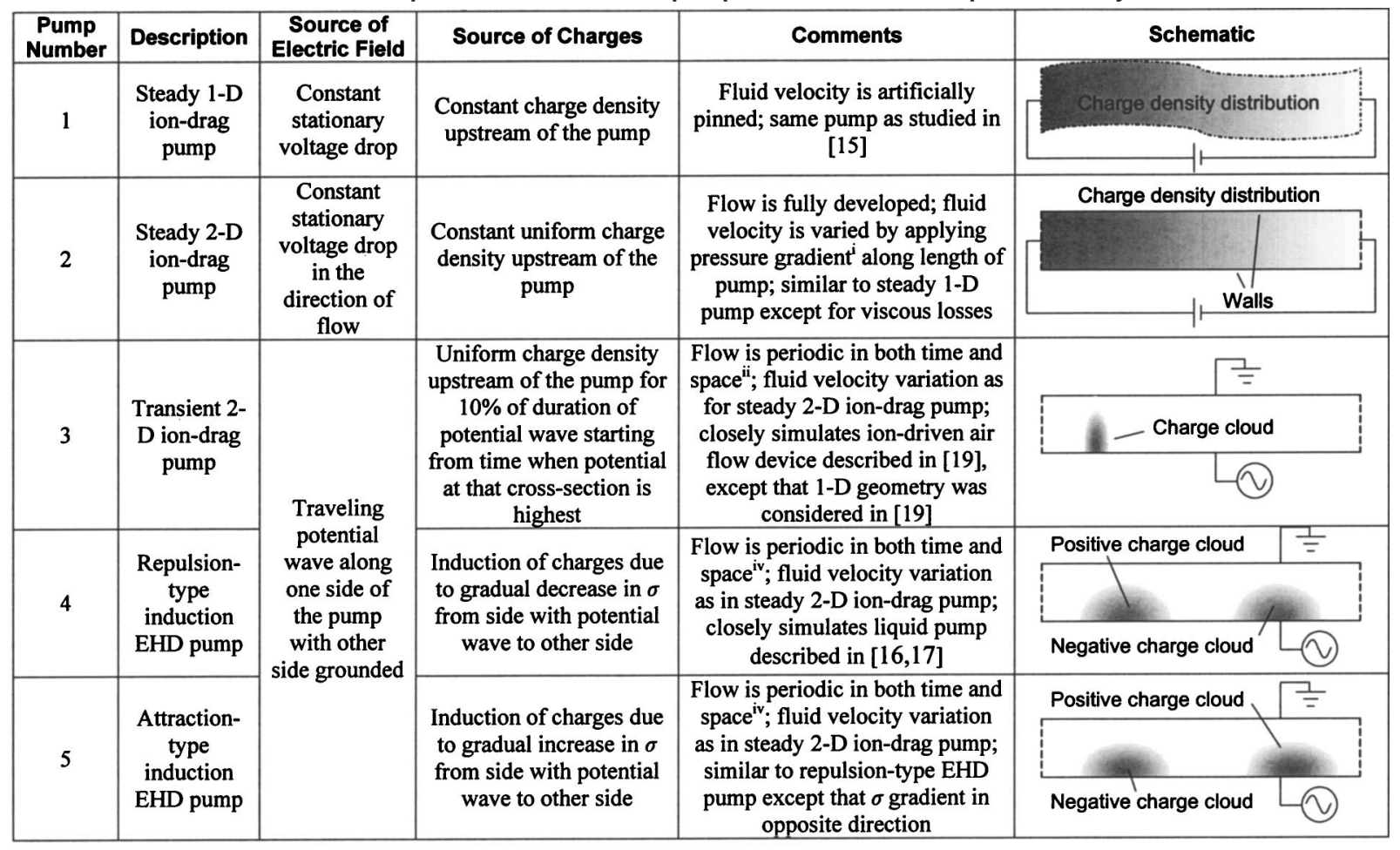

${ }^{\mathrm{i}}$ Additional to pressure head generated by EHD

ii Time periodicity: Same velocity profile repeated at every cross-section after a time equal to the time-period of the potential wave Space periodicity: At any given time, same velocity profile repeated after a distance equal to the wavelength of the potential wave

$$
P_{o}=\int\left(\left(-p \delta_{i j}+\mu_{\mathrm{vis}}\left(v_{i, j}+v_{j, i}\right)\right) \hat{j} \cdot \mathbf{v}\right)_{\mathrm{EHD}}-\left\{\left[-p \delta_{i j}+\mu_{\mathrm{vis}}\left(v_{i, j}+v_{j, i}\right)\right] \hat{j} \cdot \mathbf{v}\right\}_{\mathrm{No}} \mathrm{EHD} d S
$$

In Cartesian coordinates, for a boundary along the $x$ direction, Eq. (18) reduces to

$$
\begin{aligned}
P_{o}= & \int\left\{-p v_{x}+\mu_{\mathrm{vis}}\left[2 v_{x, x} v_{x}+\left(v_{x, y}+v_{y, x}\right) v_{y}+\left(v_{x, z}+v_{z, x}\right) v_{z}\right]\right\}_{\mathrm{EHD}}-\left\{-p v_{x}+\mu_{\mathrm{vis}}\left[2 v_{x, x} v_{x}+\left(v_{x, y}+v_{y, x}\right) v_{y}+\left(v_{x, z}\right.\right.\right. \\
& \left.\left.\left.+v_{z, x}\right) v_{z}\right]\right\}_{\mathrm{No} \mathrm{EHD}} d S_{x}
\end{aligned}
$$

Similar expressions may be written for the $y$ and $z$ directions.

The efficiency of the EHD pump is the ratio of mechanical power output to electrical power input. From Eqs. (15) and (18), this efficiency can be written as

$$
\eta_{\mathrm{E} \ell}=\frac{P_{o}}{P_{i}}=\frac{\int\left(\left(-p \delta_{i j}+\mu_{\mathrm{vis}}\left(v_{i, j}+v_{j, i}\right)\right) \hat{j} \cdot \mathbf{v}\right)_{\mathrm{EHD}}-\left\{\left[-p \delta_{i j}+\mu_{\mathrm{vis}}\left(v_{i, j}+v_{j, i}\right)\right] \hat{j} \cdot \mathbf{v}\right\}_{\mathrm{No} \mathrm{EHD}} d S}{\int \mathbf{E} \cdot(\sigma \mathbf{E}+q \mathbf{v}+q \mu \mathbf{E}) d V o l}
$$

For two-dimensional EHD pumps, the above equation can be written in Cartesian coordinates as follows

$$
\begin{gathered}
\int\left(-p v_{x}+\mu_{\mathrm{vis}}\left(2 v_{x, x} v_{x}+\left(v_{x, y}+v_{y, x}\right) v_{y}\right)\right)_{\mathrm{EHD}}-\left(-p v_{x}+\mu_{\mathrm{vis}}\left(2 v_{x, x} v_{x}+\left(v_{x, y}+v_{y, x}\right) v_{y}\right)\right)_{\text {No EHD }} d y \\
\eta_{\mathrm{E} \ell}=\frac{+\int\left\{-p v_{y}+\mu_{\mathrm{vis}}\left[2 v_{y, y} v_{y}+\left(v_{y, x}+v_{x, y}\right) v_{x}\right]\right\}_{\mathrm{EHD}}-\left(-p v_{y}+\mu_{\mathrm{vis}}\left(2 v_{y, y} v_{y}+\left(v_{y, x}+v_{x, y}\right) v_{x}\right)\right)_{\text {No EHD }} d x}{\iint\left[(\sigma+q \mu)\left(E_{x}^{2}+E_{y}^{2}\right)+q\left(E_{x} v_{x}+E_{y} v_{y}\right)\right] d x d y}
\end{gathered}
$$

For each of the different pumps considered in this work, this equation reduces to the simplified versions developed below. The five pumps considered are described with the help of schematic diagrams in Table 1. It may be noted that in the following three equations, flow without EHD is due to a constant one-dimensional pressure gradient. 
One-dimensional ion-drag pump: $\quad \eta_{\mathrm{E} \ell}=\frac{\left(p v_{x}\right)_{\mathrm{No} \mathrm{EHD}}-\left(p v_{x}\right)_{\mathrm{EHD}}}{\int\left[(\sigma+q \mu) E_{x}^{2}+q E_{x} v_{x}\right] d x}$

Two-dimensional ion-drag pump due to a stationary one-dimensional potential gradient:

$$
\eta_{\mathrm{E} \ell}=\frac{\int\left(\left(p v_{x}\right)_{\mathrm{No} \mathrm{EHD}}-\left(p v_{x}\right)_{\mathrm{EHD}}\right) d y+\int\left(\mu_{\mathrm{vis}} v_{x, y} v_{x}\right)_{\mathrm{EHD}}-\left(\mu_{\mathrm{vis}} v_{x, y} v_{x}\right)_{\mathrm{No} \mathrm{EHD}} d x}{\iint\left[(\sigma+q \mu) E_{x}^{2}+q E_{x} v_{x}\right] d x d y}
$$

Two-dimensional ion-drag pump due to a traveling potential wave, and two-dimensional attraction- and repulsion-type EHD induction pumps:

$$
\eta_{\mathrm{E} \ell}=\frac{\int\left[\left(p v_{x}\right)_{\mathrm{No} \mathrm{EHD}}-\left(p v_{x}\right)_{\mathrm{EHD}}\right] d y+\int\left(\mu_{\mathrm{vis}} v_{x, y} v_{x}\right)_{\mathrm{EHD}}-\left(\mu_{\mathrm{vis}} v_{x, y} v_{x}\right)_{\mathrm{No} \mathrm{EHD}} d x+\int\left\{\mu_{\mathrm{vis}}\left[2 v_{x, x} v_{x}+\left(v_{x, y}+v_{y, x}\right) v_{y}\right]\right\}_{\mathrm{EHD}} d y+\int\left[-p v_{y}+\mu_{\mathrm{vis}}\left(2 v_{y, y} v_{y}+v_{y, x} v_{x}\right)\right]_{\mathrm{EHD}} d x}{\iint\left[(\sigma+q \mu)\left(E_{x}^{2}+E_{y}^{2}\right)+q\left(E_{x} v_{x}+E_{y} v_{y}\right)\right] d x d y}
$$

Overall, the efficiency of the flow generation due to several actuating mechanisms which may include both electrical and mechanical forces would be given by the ratio of total fluid power output to total power input. This efficiency can be written as

$$
\eta_{\mathrm{tot}}=\frac{P_{o, \mathrm{EHD}}}{P_{i, \mathrm{E} \ell}+P_{i, m}}=\frac{\int\left(\left(-p \delta_{i j}+\mu_{\mathrm{vis}}\left(v_{i, j}+v_{j, i}\right)\right) \hat{j} \cdot \mathbf{v}\right)_{\mathrm{EHD}} d S}{\int \mathbf{E} \cdot(\sigma \mathbf{E}+q \mathbf{v}+q \mu \mathbf{E}) d V o l+\int\left(p_{\mathrm{app}} \delta_{i j}\right) \hat{j} \cdot \mathbf{v}_{\mathrm{EHD}} d S}
$$

In two-dimensional Cartesian coordinates, this may be written as

$$
\eta_{\mathrm{tot}}=\frac{\int\left\{-p v_{x}+\mu_{\mathrm{vis}}\left[2 v_{x, x} v_{x}+\left(v_{x, y}+v_{y, x}\right) v_{y}\right]\right\}_{\mathrm{EHD}} d y+\int\left(-p v_{y}+\mu_{\mathrm{vis}}\left(2 v_{y, y} v_{y}+\left(v_{y, x}+v_{x, y}\right) v_{x}\right)\right)_{\mathrm{EHD}} d x}{\iint\left[(\sigma+q \mu)\left(E_{x}^{2}+E_{y}^{2}\right)+q\left(E_{x} v_{x}+E_{y} v_{y}\right)\right] d x d y+\int p_{\mathrm{app}} v_{x, \mathrm{EHD}} d y+\int p_{\mathrm{app}} v_{y, \mathrm{EHD}} d x}
$$

Equation (26) can be applied to each of the pump designs considered to obtain simplified expressions for $\eta_{\text {tot }}$. It is noted that $\eta_{\text {tot }}$ is the efficiency of the complete fluidic system, which may include both electrical and mechanical actuating forces. On the other hand, $\eta_{\mathrm{E} \ell}$ is the efficiency of solely the electrical forces in causing fluid motion. However, $\eta_{\mathrm{E} \ell}$ is not independent of mechanical forces as the change in bulk fluid velocity due to mechanical forces affects $\eta_{\mathrm{E} \ell}$

Numerical Modeling. The commercially available computational fluid dynamics software package FIDAP was used for numerical modeling [18]. Flow was assumed to be laminar for all the cases considered. A built-in EHD module in FIDAP was used along with user-defined subroutines developed for calculating the input electrical power and output fluidic power. Validation of the iondrag and induction EHD models is reported in detail [16], where the ion-drag EHD model was validated by comparison against the results of [15], while the induction EHD model was validated by comparison to [19]. The geometry was modeled using secondorder elements. Picard iteration method was used to solve the discretized equations. Mesh-independence tests were performed for potential, charge density, and flow velocity. On doubling the number of elements in each direction, the values of these parameters varied less than $1 \%$ for steady-state simulations and less than $2 \%$ for transient simulations. Time stepping was done dynamically using the trapezoidal rule, which is a second-order implicit time-integration scheme with maximum relative local time truncation error of $0.1 \%$.
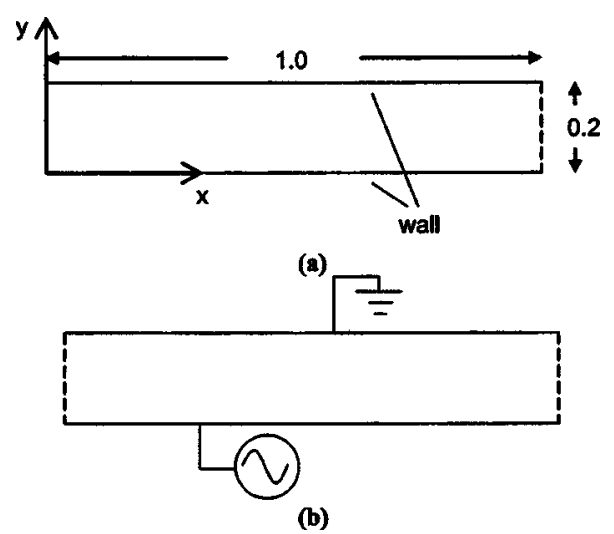

Fig. 1 (a) Domain under consideration in two-dimensional pumps; (b) potential wave application in pumps with traveling potential wave

All computations were performed with the following set of parameters. A fluid with $\varepsilon=\sigma=\mu=\mu_{\mathrm{vis}}=1$ was considered. The twodimensional domain under consideration in pumps 2-5 (pump numbers identified in Table 1) is shown in Fig. 1. The length of the domain was $L=1$ for all pumps, and the width was $w=0.2$ for the two-dimensional pumps. These parameter choices help simplify the nondimensional parameters governing the problem to 
Table 2 Comparison of $\eta$ values for a 1D EHD pump obtained from the present model to values from the literature [15].

\begin{tabular}{|c|c|c|c|c|}
\hline \multirow{2}{*}{$R_{\theta_{\text {e }}}$} & \multirow{2}{*}{$E_{s}$} & Esl & \multicolumn{2}{|c|}{ n(Eficiency) } \\
\cline { 4 - 5 } & & & Present work & $\begin{array}{c}\text { Results from } \\
{[15]}\end{array}$ \\
\hline 200 & 1 & 0 & 0.98 & 0.98 \\
\hline 200 & 1 & 0.1 & 0.89 & 0.89 \\
\hline 200 & 1 & 1 & 0.49 & 0.49 \\
\hline 200 & 1 & 10 & 0.09 & 0.09 \\
\hline 100 & 0.1 & 0 & 0.90 & 0.90 \\
\hline 100 & 0.1 & 0.1 & 0.83 & 0.83 \\
\hline 100 & 0.1 & 1 & 0.47 & 0.47 \\
\hline 100 & 0.1 & 10 & 0.09 & 0.09 \\
\hline
\end{tabular}

$\operatorname{Re}_{\mathrm{E} \ell}=v, E s l=V_{e} / v$, and $E s=q_{e} / V_{e}$. Also, the units for all the parameter values listed here need only follow a consistent framework, such as those mentioned in the Nomenclature. The results would be valid for any consistent set of units. While the results depend on the choice of these parameter values, the focus here is on the trends of variation obtained.

For the pumps under steady operation (pumps 1 and 2), $\Phi(x$ $=0, y)=q(x=0, y)=100$ and $\Phi(x=1, y)=0$. For the pumps in transient operation (pumps 3-5), the initial potential and charge density were zero throughout the domain, i.e., $\Phi(x, y, t=0)=0$ $=q(x, y, t=0)$. The potential wave at the electrode wall for all transient pumps was characterized by $\Phi=100, \omega=k=2 \pi$, i.e., $\Phi(x, y=0)=100 \cos (2 \pi t-2 \pi x)$. The other wall was grounded, i.e., $\Phi(x, y=0.2)=0$. For pump $3, q=100$ at $x=0$ for $10 \%$ of the potential wave duration, i.e., $q(x=0, y)=\left\{\begin{array}{c}100,0 \leqslant t<0.1 / 2 \pi \\ 0,0.1 / 2 \pi \leqslant t<1 / 2 \pi\end{array}\right.$. The constant conductivity gradient for charge induction in pump 4 can be characterized by $\Delta \sigma=\sigma(x, y=0)-\sigma(x, y=0.2)=0.1$, while for pump $5 \Delta \sigma=\sigma(x, y=0)-\sigma(x, y=0.2)=-0.1$.

Efficiency values calculated from the present model are compared in Table 2 for several different cases to values read from graphs in [15]; the efficiency of a one-dimensional EHD pump with an applied voltage difference across the domain and a constant charge density upstream of the domain was reported in [15]. The two sets of results, presented in terms of $\mathrm{Re}_{\mathrm{E} \ell}, E s$ and $E s l$, are seen to be identical.

\section{Results and Discussion}

For each of the five pumps considered in this work (as in Table 1), results are presented in terms of the efficiency obtained with EHD action alone, as well as with the combined action of EHD and an external pressure gradient. Efficiency values are presented as a function of the nondimensional average bulk fluid velocity along the length of pump, defined as $v_{\mathrm{fl}}^{*}=v_{\mathrm{fl}} / v_{\mathrm{fl}, E}$, where $v_{\mathrm{fl}}$ is the fluid velocity due to combined action of EHD and pressure gradient and $v_{\mathrm{fl}, E}$ is the fluid velocity due only to the EHD action without any external pressure gradient. This is a more suitable parameter for examining the effect of bulk velocity on pump efficiency since both Reynolds number $\left(\operatorname{Re}=\rho v_{\mathrm{fl}} h / \mu_{\mathrm{vis}}\right)$ and Electric Reynolds number $\left(\operatorname{Re}_{\mathrm{E} \ell}=\varepsilon v / \sigma L\right)$ involve parameters which could independently change the results without a change in the bulk fluid velocity. In addition to the efficiency results, variations with bulk fluid velocity of the nondimensional total input power $\left(P_{i, \text { tot }}^{*}\right.$, which includes both the electrical input power and mechanical input power used to create the pressure gradient), total output power due to combined action of EHD and external pressure gradient $\left(P_{o, \text { tot }}^{*}\right)$, electrical input power $\left(P_{i, \mathrm{E} \ell}^{*}\right)$, and fluidic output power solely due to $\operatorname{EHD}\left(P_{o, \mathrm{E} \ell}^{*}\right.$, power transferred to the fluid due to EHD) are also presented. Both the total and the electrical input power as well as the total and the electrical output power are nondimensionalized by the electrical output power in

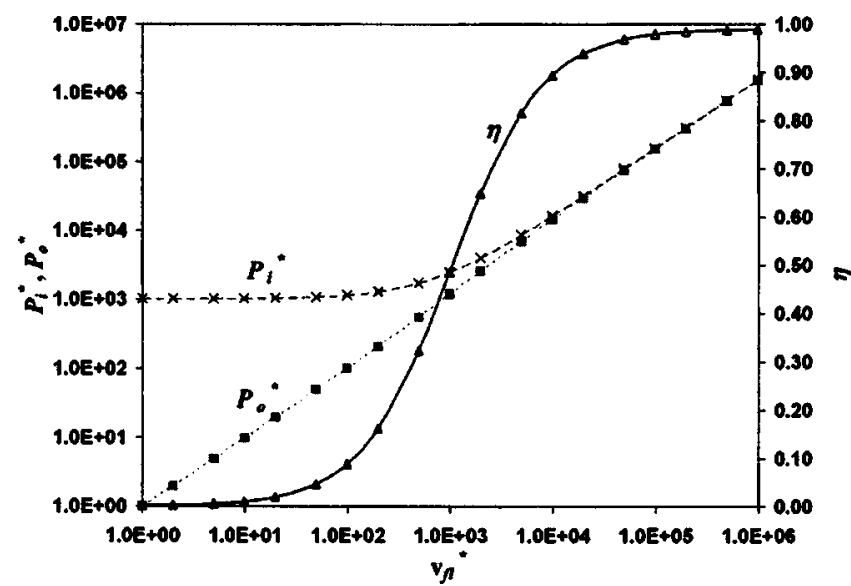

Fig. 2 Effect of variation of nondimensional bulk fluid velocity on nondimensional input power, output power, and efficiency for the steady one-dimensional ion-drag pump

the absence of an external pressure gradient $\left(P_{o, E}\right)$, i.e., $P_{i, \text { tot }}^{*}$ $=P_{i, \mathrm{tot}} / P_{o, E}, \quad P_{i, \mathrm{E} \ell}^{*}=P_{i, \mathrm{E} \ell} / P_{o, E}, \quad P_{o, \text { tot }}^{*}=P_{o, \text { tot }} / P_{o, E} \quad$ and $\quad P_{o, \mathrm{E} \ell}^{*}$ $=P_{o, \mathrm{E} \ell} / P_{o, E}$.

One-Dimensional Steady-State Ion-Drag Pump. The variation of nondimensional input and output power $\left(P_{i}^{*}\right.$ and $\left.P_{o}^{*}\right)$ and efficiency $(\eta)$ with nondimensional bulk fluid velocity $\left(v_{\mathrm{fl}}^{*}\right)$ for a 1D pump is shown in Fig. 2. All input parameters except for $v_{\mathrm{fl}}^{*}$ are held constant. The horizontal axis $\left(v_{\mathrm{fl}}^{*}\right)$ and left vertical axis $\left(P_{i}^{*}\right.$ and $\left.P_{o}^{*}\right)$ have a logarithmic variation. The right vertical axis $(\eta)$ has a linear variation. It may be noted that in a $1 \mathrm{D}$ system, the velocity needs to be artificially pinned. Therefore, $P_{i}, P_{o}$, and $v_{\mathrm{fl}}$ are nondimensionalized with respect to the corresponding values at the lowest $v_{\mathrm{fl}}$ considered.

Figure 2 shows that $P_{i}^{*}, P_{o}^{*}$ and $\eta$ increase with an increase in $v_{\mathrm{fl}}^{*}$. This can be explained as follows. As $v_{\mathrm{fl}}^{*}$ increases, the current due to charge convection increases and hence the input electrical power $P_{i}^{*}$ increases. Moreover, since current due to charge conduction and mobility does not vary much with $v_{\mathrm{fl}}^{*}$, the ratio of convection current to total current increases according to Eq. (15). The conversion of electrical power to fluidic power is most efficient for convection current because there is no charge decay as is the case for charge conduction and mobility. This causes an increase in the efficiency of EHD action. Increases in both $P_{i}^{*}$ and $\eta$ lead to the increase in $P_{o}^{*}$ seen in Fig. 2. Since all the electrical and mechanical parameters except for bulk fluid velocity are fixed, this increase in efficiency is solely due to the increase in $v_{\mathrm{fl}}$. It may be noted that no distinction is made between $P_{i, \text { tot }}, P_{o \text {,tot }}$, $\eta_{\mathrm{tot}}$, and $P_{i, \mathrm{E} \ell}, P_{o, \mathrm{E} \ell}, \eta_{\mathrm{E} \ell}$ because $1 \mathrm{D}$ flow does not offer any pressure gradient or wall friction. Hence subscripts "tot" and "El" have been dropped from parameters $P_{i}, P_{o}$, and $\eta$ here.

The 1D case considered above is clearly an idealized situation. It can be thought to represent the ideal operation of an ion-drag EHD pump. Since there is no pressure gradient to be overcome, any pressure head generated by EHD will increase the fluid velocity infinitely unless it is limited by some external means. In the present simulations, these velocities were limited using the inlet velocity boundary condition. A more practical situation would involve flow created using EHD in a pipe or over a plate. In that case, EHD would need to overcome frictional forces at the surface, which would naturally limit the fluid velocity. Such more realistic pumps, with fully developed flow between parallel plates due to differing voltage and charge densities, are considered in the following.

Two-Dimensional Steady-State Ion-Drag Pump. The varia- 


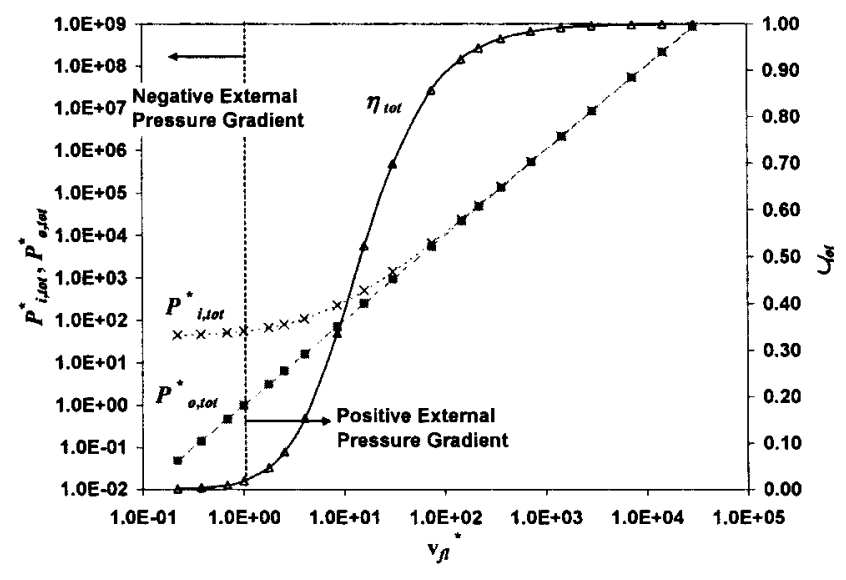

Fig. 3 Variation of nondimensional total input power, total output power, and total with nondimensional bulk fluid velocity due to combined action of EHD in the steady two-dimensional ion-drag pump and varying external pressure gradient

tion of nondimensional total input power $P_{i, \text { tot }}^{*}$, total output power $P_{o \text {,tot }}^{*}$ and total efficiency $\eta_{\text {tot }}$ with nondimensional average bulk fluid velocity $v_{\mathrm{fl}}^{*}$ for this pump is shown in Fig. 3. Again the horizontal axis $\left(v_{\mathrm{fl}}^{*}\right)$ and left vertical axis $\left(P_{i, \text { tot }}^{*}\right.$ and $\left.P_{o \text {,tot }}^{*}\right)$ have logarithmic variations, while the right vertical axis $\left(\eta_{\text {tot }}\right)$ has a linear variation. The velocity $v_{\mathrm{fl}}^{*}$ is varied by applying an external pressure gradient in addition to the EHD action. Both negative and positive pressure gradients are considered. Results for the negative pressure gradient are reported only for cases for which there is still a net flow in the direction of EHD pumping.

For this pump, $P_{i, \text { tot }}^{*}$ and $P_{o \text {,tot }}^{*}$ are seen to follow the same trend of variation as for the one-dimensional pump. The monotonic rise in total efficiency $\eta_{\text {tot }}$ with $v_{\mathrm{fl}}^{*}$ can be attributed to two reasons. A positive pressure gradient causes additional flow in the forward direction, which increases charge convection and hence the output fluid power due to EHD, as was explained above. More importantly for the present pump, a pressure gradient creates flow with perfect efficiency and hence as the ratio of power input due to the pressure gradient to power input from EHD increases, the overall rectification efficiency increases. While the general trend of variation of efficiency for the present pump is similar to that of the previous pump considered, $P_{o \text {,tot }}^{*}$ varies roughly as the square of $v_{\mathrm{fl}}^{*}$. This is expected since the output power from the external pressure gradient varies as square of the pressure gradient $\left(P_{m}\right.$ $\propto p^{2}$ ) while the fluid velocity varies linearly with pressure gradient $\left(v_{\mathrm{fl}} \propto p\right)$; thus, output power from the external pressure gradient is proportional to the square of fluid velocity $\left(P_{m} \propto v_{\mathrm{fl}}^{2}\right)$.

The electrical contributions to the input and output power, as well as the efficiency, $P_{i, \mathrm{E} \ell}^{*}, P_{o, \mathrm{E} \ell}^{*}$ and $\eta_{\mathrm{E} \ell}$ are plotted as a function of $v_{\mathrm{fl}}^{*}$ in Fig. 4. Again, $v_{\mathrm{fl}}^{*}, P_{i, \mathrm{E} \ell}^{*}$ and $P_{o, \mathrm{E} \ell}^{*}$ are plotted on a logarithmic scale and $\eta_{\mathrm{E} \ell}$ is on a linear scale. The results are plotted only for cases when the net flow is in the positive direction. In this figure, $\eta_{\mathrm{E} \ell}$ represents the actual efficiency of the EHD action. While it remains rather small relative to $\eta_{\text {tot }}$ (shown in Fig. 3), $\eta_{\mathrm{E} \ell}$ increases from less than 0.02 (2\% efficient) for no external pressure gradient to more than $0.19(19 \%$ efficient $)$ for $v_{\mathrm{fl}}^{*}=147$; at this velocity, $\eta_{\text {tot }}$ is 0.92 . This increase in $\eta_{\mathrm{E} \ell}$ is due to the combined effect of an increase in $P_{i, \mathrm{E} \ell}^{*}$ and a sharper increase in $P_{o, \mathrm{E} \ell}^{*}$, both due to increased charge convection, as was explained in the case of the one-dimensional pump.

Figure 4 also shows that the output fluid power solely due to EHD action, $P_{o, \mathrm{E} \ell}^{*}$, increases in the presence of the positive external pressure gradient. This implies that the net fluid power achieved from the combined action of EHD and external pressure gradient is greater than the sum of the fluid powers achieved from

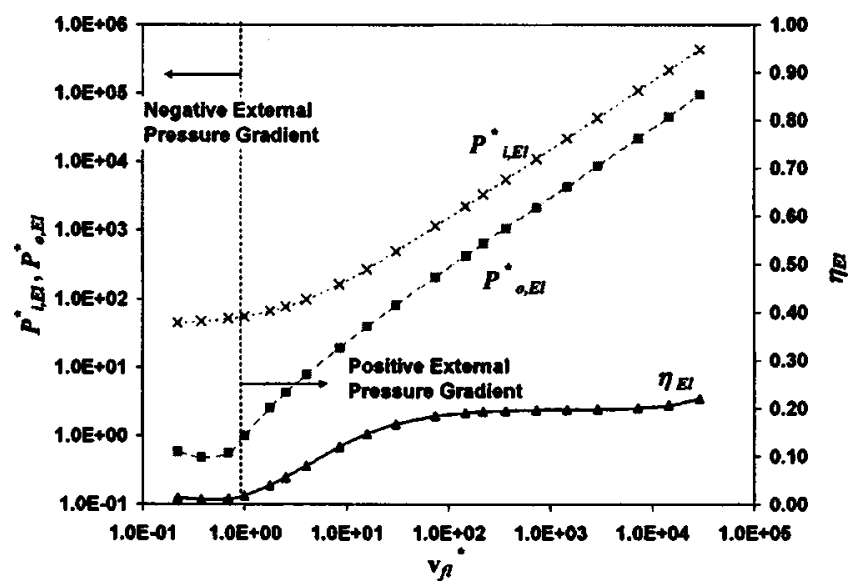

Fig. 4 Variation of nondimensional electrical input power, output power due to EHD, and efficiency due to EHD with nondimensional bulk fluid velocity due to combined action of EHD in the steady two-dimensional ion-drag pump and varying external pressure gradient

their action independent of each other.

The quantity $P_{o, \mathrm{E} \ell}^{*}$ is the ratio of output fluid power solely due to EHD to the input electrical power; output fluid power is calculated by subtracting the mechanical fluid power due to the pressure gradient from that due to the combined action of EHD and pressure gradient, i.e., $P_{o, \mathrm{E} \ell}^{*}=\left(P_{o, \mathrm{tot}}-P_{o, m}\right) / P_{o, \mathrm{EHD}}$. At larger values of $v_{\mathrm{fl}}^{*}$, both $P_{o \text {,tot }}$ and $P_{o, m}$ can be several orders of magnitude larger than $P_{o, \mathrm{EHD}}$, while $\left(P_{o, \text { tot }}-P_{o, m}\right)$ is of the same order of magnitude as $P_{o \text {,EHD }}$. Hence even small numerical inaccuracies in either $P_{o, \text { tot }}$ or $P_{o, m}$ can result in large discrepancies in the values of $P_{o, \mathrm{E} \ell}^{*}$ and $\eta_{\mathrm{E} \ell}$. The increase in rate of variation of $\eta_{\mathrm{E} \ell}$ for $v_{\mathrm{fl}}^{*}$ $>10^{4}$ in Fig. 4 is believed to be due to these numerical inaccuracies.

Results for $P_{o, \mathrm{E} \ell}$ such as those presented above may be employed to generate a pump curve for the system, as is done in the following for each of the two-dimensional pumps considered. The pump curve for the two-dimensional steady-state ion-drag pump is shown in Fig. 5. The inset is a magnified view for small values of $v_{\mathrm{fl}}^{*}$. The nondimensional pressure head generated by the pump, $P_{\mathrm{E} \ell}^{*}$, has been obtained using the following expression

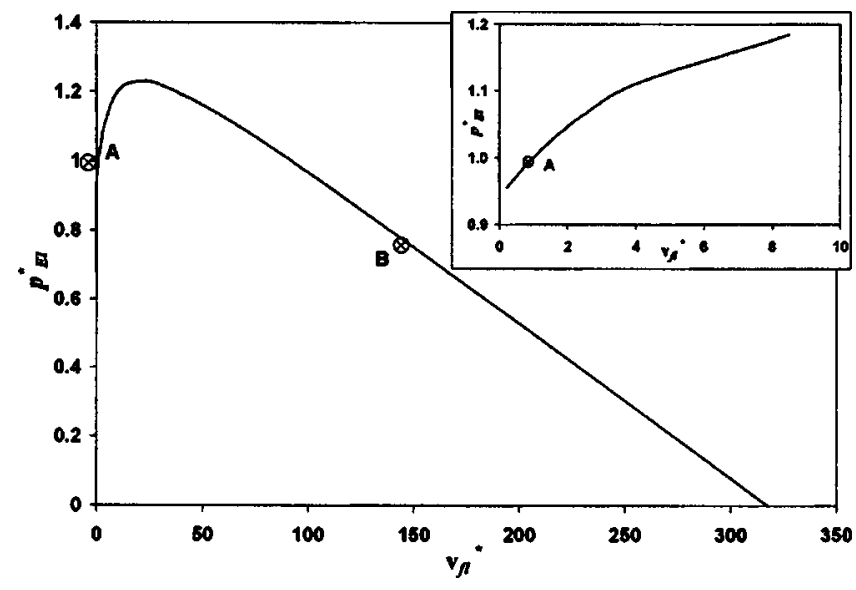

Fig. 5 Nondimensional pump curve for the steady twodimensional ion-drag pump. (Inset: Magnified view of pump curve for small values of nondimensional bulk fluid velocity.) 


$$
p_{\mathrm{E} \ell}^{*}=\frac{p_{\mathrm{E} \ell}}{p_{\mathrm{EHD}}}=\frac{\left(\int \mu_{\mathrm{vis}} v_{x, y} v_{x} d x-\int\left(p+p_{\mathrm{app}}\right) v_{x} d y\right) / v_{x}}{\left[\left(\int \mu_{\mathrm{vis}} v_{x, y} v_{x} d x-\int p v_{x} d y\right) / v_{x}\right]_{\mathrm{EHD}}}
$$

Here, $p$ is the pressure head generated by EHD and $p_{\text {app }}$ is applied external pressure gradient. When $p_{\text {app }}=0$, the EHD pump operates at $v_{\mathrm{fl}}^{*}=1, p_{\mathrm{E} \ell}^{*}=1$, which is marked as point $\mathrm{A}$ in Fig. 5. It is clear that this is not the best operating point for the EHD pump. The maximum output of the pump $\left(v_{\mathrm{fl}}^{*} \times p_{\mathrm{E} \ell}^{*}\right)$ among the points considered is at $v_{\mathrm{fl}}^{*}=147.23, p_{\mathrm{E} \ell}^{*}=0.76$. This point is identified as point B in Fig. 5. The output of the EHD pump is more than 112 times higher at this point than at $v_{\mathrm{fl}}^{*}=1, p_{\mathrm{E} \ell}^{*}=1$, and it decreases on moving away from this point in either direction. This optimal operation at point $\mathrm{B}$ is also achieved at lower $p_{\mathrm{E} \ell}^{*}$ and higher $v_{\mathrm{fl}}^{*}$ than under conditions where the EHD pump operates without an external pressure gradient. This reinforces the conclusion that an increase in the bulk fluid velocity causes an increase in output power of the pump.

It is important to emphasize the difference between the quantities $P_{o, \mathrm{E} \ell}^{*}, P_{o \text {,tot }}^{*}$ and $v_{\mathrm{fl}}^{*} \times p_{\mathrm{E} \ell}^{*}$. The first of these, $P_{o, \mathrm{E} \ell}^{*}$, is a measure of the increase in output fluid power due to operation of the EHD pump; $P_{o \text {,tot }}^{*}$, on the other hand, is a measure of the total output fluid power due to the EHD pump and external pressure gradient. In contrast, $v_{\mathrm{fl}}^{*} \times p_{\mathrm{E} \ell}^{*}$ is a measure of the net output fluidic power of the EHD pump. It represents how the pump would operate under different hydrodynamic conditions. The equations for $P_{o, \mathrm{E} \ell}^{*}, P_{o \text {,tot }}^{*}$, and $v_{\mathrm{fl}}^{*} \times p_{\mathrm{E} \ell}^{*}$ are presented explicitly below to clarify their definitions; it may be noted that all three have the same denominator.

$$
P_{o, \mathrm{E} \ell}^{*}=\frac{\left(\int \mu_{\mathrm{vis}} v_{x, y} v_{x} d x-\int p v_{x} d y\right)_{\mathrm{EHD}}-\left(\int \mu_{\mathrm{vis}} v_{x, y} v_{x} d x-\int p v_{x} d y\right)_{\text {No EHD }}}{\left(\int \mu_{\mathrm{vis}} v_{x, y} v_{x} d x-\int p v_{x} d y\right)_{E}}
$$

$$
\begin{aligned}
P_{o, \text { tot }}^{*} & =\frac{\left(\int \mu_{\mathrm{vis}} v_{x, y} v_{x} d x-\int p v_{x} d y\right)_{\mathrm{EHD}}}{\left(\int \mu_{\mathrm{vis}} v_{x, y} v_{x} d x-\int p v_{x} d y\right)_{E}} \\
v_{\mathrm{fl}}^{*} \times p_{\mathrm{E} \ell}^{*} & =\frac{\left(\int \mu_{\mathrm{vis}} v_{x, y} v_{x} d x-\int\left(p+p_{\mathrm{app}}\right) v_{x} d y\right)}{\left(\int \mu_{\mathrm{vis}} v_{x, y} v_{x} d x-\int p v_{x} d y\right)_{E}}
\end{aligned}
$$

Two-Dimensional Transient Ion-Drag Pump. Simulations for all the transient pumps were run until each pump reached a quasisteady-state operation, where the results start repeating over the time period of the potential wave. The results shown for these pumps are time averaged over one period of the potential wave after the pumps have reached this quasi-steady state.

Variation of $P_{i, \text { tot }}^{*}, P_{o, \text { tot }}^{*}$, and $\eta_{\text {tot }}[$ Fig. $6(a)], P_{i, \mathrm{E} \ell}^{*}, P_{o, \mathrm{E} \ell}^{*}$, and $\eta_{\mathrm{E} \ell}[$ Fig. $6(b)]$ and $p_{\mathrm{E} \ell}^{*}$ with $v_{\mathrm{fl}}^{*}[$ Fig. $6(c)]$ for the two-dimensional transient ion-drag pump are shown in Fig. 6 . The trends of variation of $P_{i \text {,tot }}^{*}, P_{o \text {,tot }}^{*}$, and $\eta_{\mathrm{tot}}$ with $v_{\mathrm{fl}}^{*}$ are the same as for the twodimensional steady ion-drag pump considered above. On the other hand, $P_{i, \mathrm{E} \ell}^{*}, P_{o, \mathrm{E} \ell}^{*}$, and $\eta_{\mathrm{E} \ell}$ show very different dependence on $v_{\mathrm{fl}}^{*}$. The input electrical power $P_{i, \mathrm{E} \ell}^{*}$ shows a rather small variation with $v_{\mathrm{fl}}^{*}$, and that too only at low $v_{\mathrm{fl}}^{*}$. This difference in behavior can be explained as follows. Charge is introduced upstream of the pump for a small portion $(10 \%)$ of the period of the potential wave. For the particular pump considered here, the bulk velocity of the fluid is much higher than the speed of the potential wave; hence, charge is swept out of the pump very quickly and for most of the portion of the period of the potential wave, there is little charge in the domain. The currents due to charge mobility and charge convection are thus limited. Current due to electrical conductivity, however, does not depend on external charge and does not vary. Hence the electrical power input $P_{i, \mathrm{E} \ell}^{*}$ is limited.

An interesting trend of variation of $P_{o, \mathrm{E} \ell}^{*}$ with $v_{\mathrm{fl}}^{*}$ is also seen in
Fig. $6(b)$. The small increase in $P_{o, \mathrm{E} \ell}^{*}$ when $v_{\mathrm{fl}}^{*}$ is close to but just greater than 1 is due to an increase in $P_{i, \mathrm{E} \ell}^{*}$ and $\eta_{\mathrm{E} \ell}$, which results from an increase in current due to charge convection. The subsequent decrease in $P_{o, \mathrm{E} \ell}^{*}$ follows the decrease in efficiency of EHD action: a majority of the current at high $v_{\mathrm{fl}}^{*}$ is due to charge conduction, which has very low efficiency. The slight upturn in $P_{o, \mathrm{E} \ell}^{*}$ at larger $v_{\mathrm{fl}}^{*}$ is due to the numerical inaccuracies discussed earlier. The pump curve for this pump is shown in Fig. 6(c). As in Fig. 5, the point of operation of the pump without any external pressure gradient is marked A, while that at which the fluid power output is a maximum $\left(40 \%\right.$ higher than at $\mathrm{A}$, where $\left.v_{\mathrm{fl}}^{*}=1, p_{\mathrm{E} \ell}^{*}=1\right)$ is marked B. The power output increases significantly with only a modest increase in $v_{\mathrm{fl}}^{*}$ due to the higher charge convection, and then decreases for larger values of $v_{\mathrm{fl}}^{*}$ because of the absence of Coulomb forces for a portion of the duration of pump operation.

Repulsion-Type Induction EHD Pump. Similar quantities as considered for the pumps above are plotted for a repulsion-type EHD pump in Fig. 7. The flow due to repulsion-type EHD is in a direction opposite to that of the traveling potential wave. The direction of flow is considered positive in the following discussion. Results for negative pressure gradients are presented only for cases in which the resultant flow is in the same direction as that due to EHD alone. The trend of variation for all three parameters in Fig. 7(a) is similar to the other cases considered thus far, with $P_{o, \text { tot }}^{*}$ showing a quadratic variation with $v_{\mathrm{fl}}^{*}$.

The variation of $P_{i, \mathrm{E} \ell}^{*}, P_{o, \mathrm{E} \ell}^{*}$, and $\eta_{\mathrm{E} \ell}$ with $v_{\mathrm{fl}}^{*}$ is shown in Fig. $7(b)$. It is seen that the increase in $P_{i, \mathrm{E} \ell}^{*}$ with increasing $v_{\mathrm{fl}}^{*}$ is very slight in this case when compared to the steady-state pumps. This is because charge induction results in the creation of equal amounts of negative and positive charges so that the net charge due to induction is zero. An increase in $v_{\mathrm{fl}}^{*}$ causes an increase in convection of both positive and negative charges and hence there is no change in the convection current. Despite little variation in $P_{i, \mathrm{E} \ell}^{*}$, however, the increase in $P_{o, \mathrm{E} \ell}^{*}$ with $v_{\mathrm{fl}}^{*}$ is significant: $P_{o, \mathrm{E} \ell}^{*}$ increases rapidly at small $v_{\mathrm{fl}}^{*}$, while the rate of increase drops off at larger $v_{\mathrm{fl}}^{*}$. The efficiency $\eta_{\mathrm{E} \ell}$ follows the same trend of variation as $P_{o, \mathrm{E} \ell}^{*}$, since there is little variation in $P_{i, \mathrm{E} \ell}^{*}$. 


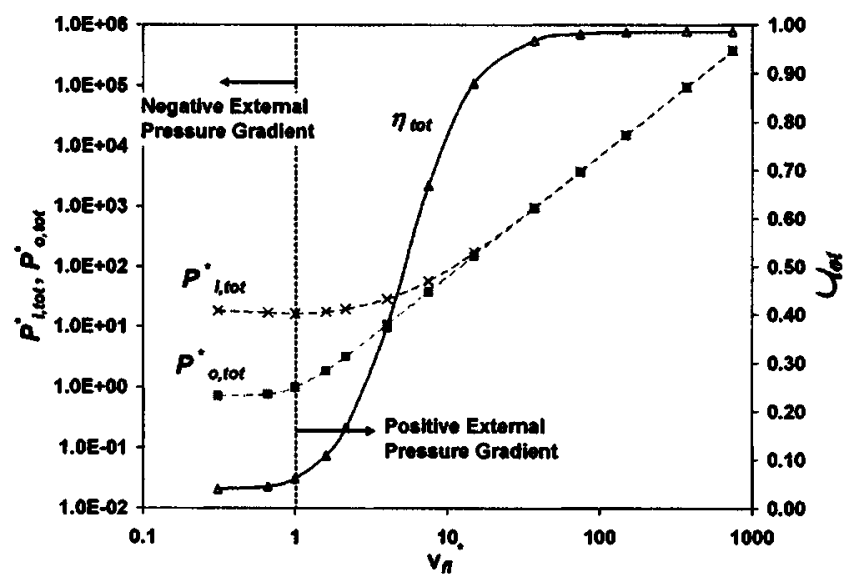

(a)

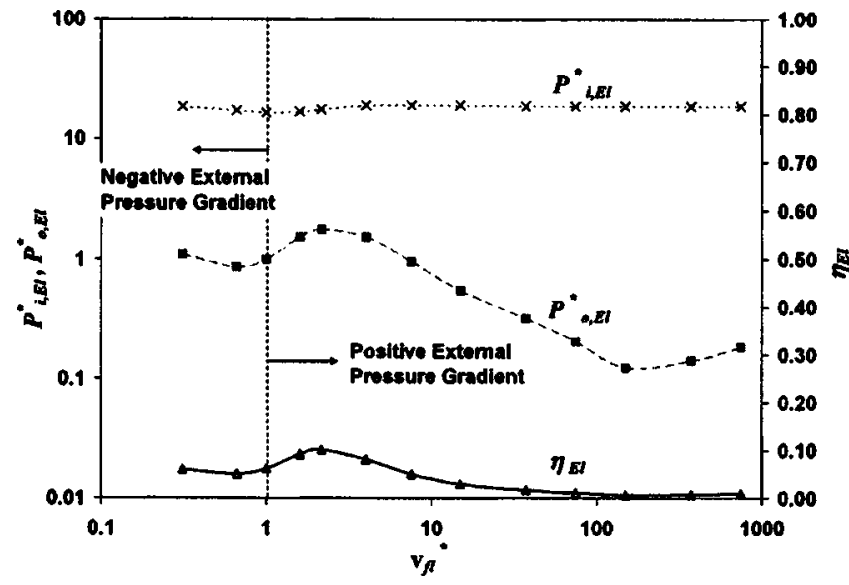

(b)

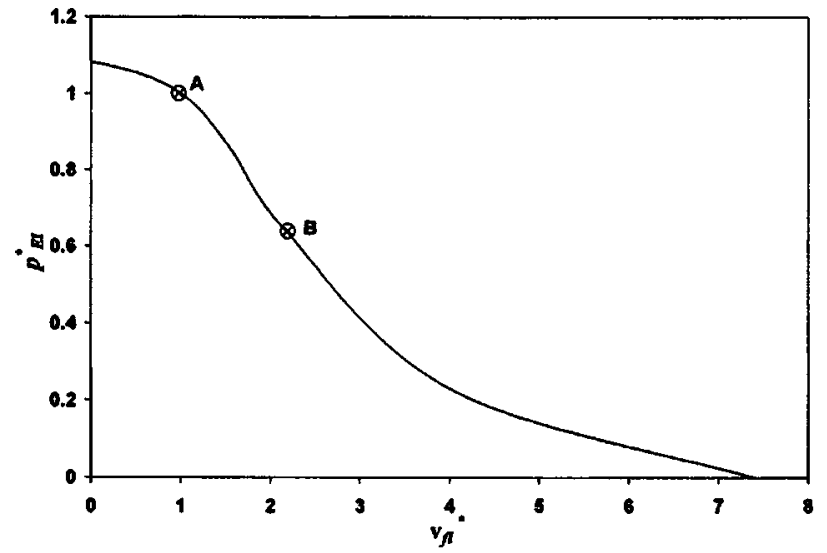

(c)

Fig. 6 Variation of (a) nondimensional total input power, total output power, and total efficiency; (b) nondimensional electrical input power, output power due to EHD, and efficiency due to EHD; and (c) pump curve, i.e., nondimensional pressure head generated by the pump with nondimensional bulk fluid velocity due to combined action of EHD in the transient twodimensional ion-drag pump and varying external pressure gradient

The total fluid power output by the combined action of EHD and pressure gradient for $v_{\mathrm{fl}}^{*}>1$ is higher than the sum of the fluid power outputs from EHD and pressure gradient when operated individually. Moreover, this difference increases with an increase in $v_{\mathrm{fl}}^{*}$. This observation is similar to that made for the steady two-dimensional ion-drag pump above.

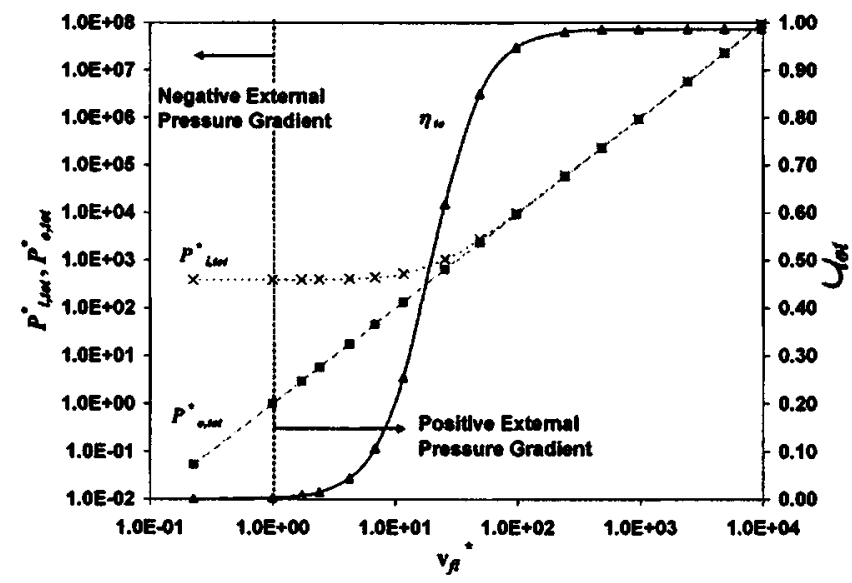

(a)

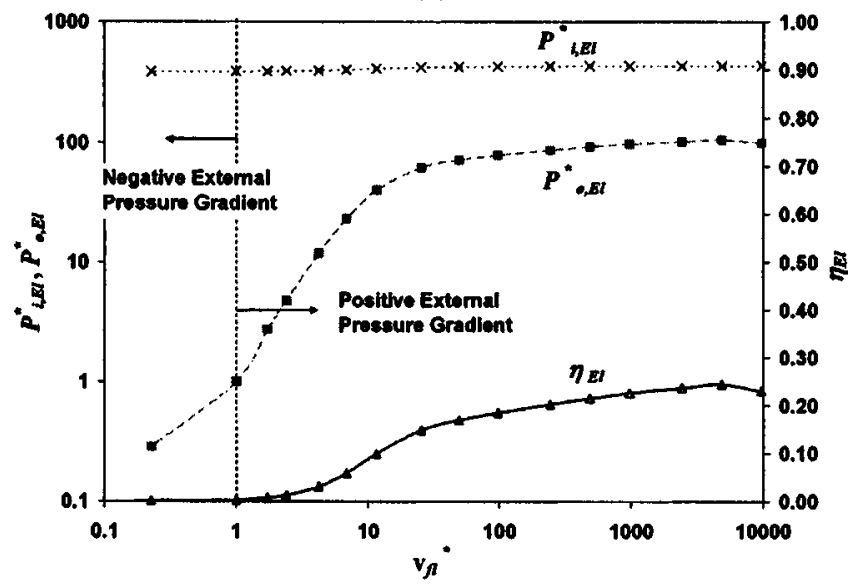

(b)

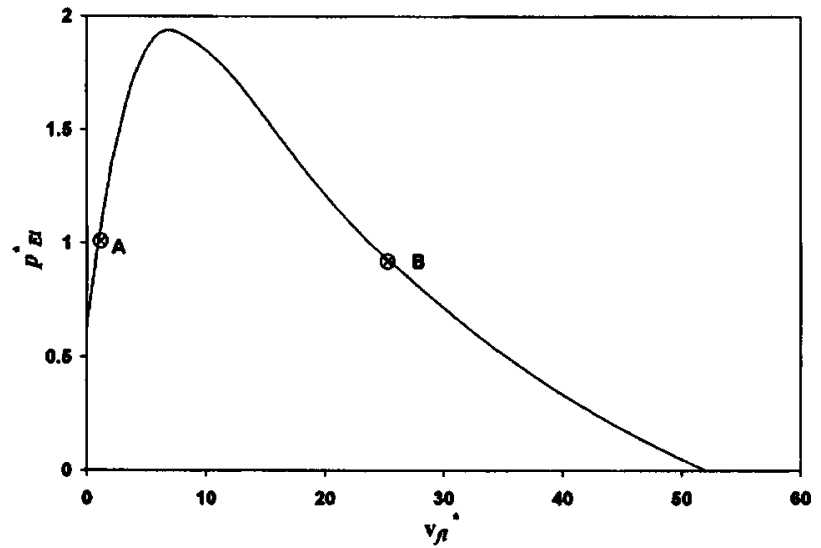

(c)

Fig. 7 Variation of (a) nondimensional total input power, total output power, and total efficiency; (b) nondimensional electrical input power, output power due to EHD, and efficiency due to EHD; and (c) pump curve, i.e., nondimensional pressure head generated by the pump with nondimensional bulk fluid velocity due to combined action of the repulsion-type induction EHD pump and varying external pressure gradient

The pump curve (variation of $p_{\mathrm{E} \ell}^{*}$ with $v_{\mathrm{fl}}^{*}$ ) for this pump is shown in Fig. 7(c). The shape of the pump curve is similar to that for the steady two-dimensional ion-drag pump. The fluid output power at its maximum (point B) is approximately 24 times that when there is no external pressure gradient (point A).

Attraction-Type Induction EHD Pump. Results for the last of 


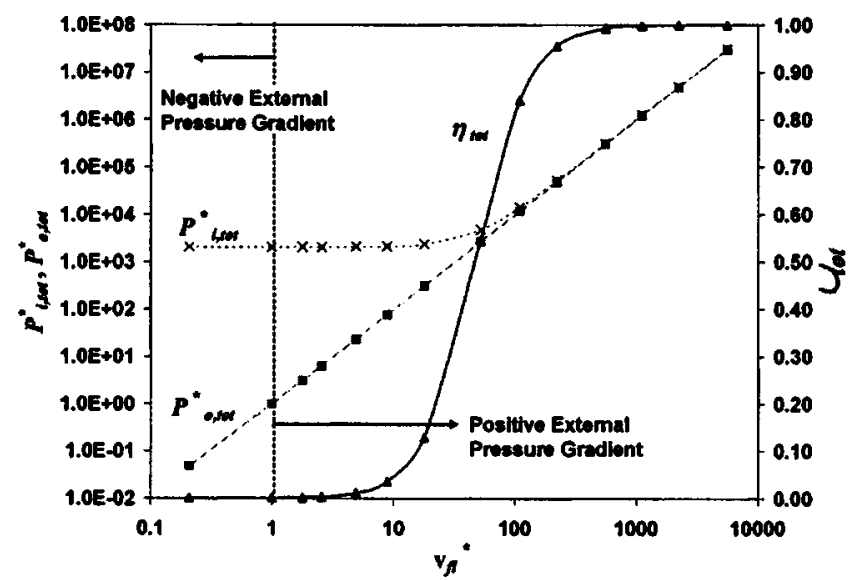

(a)

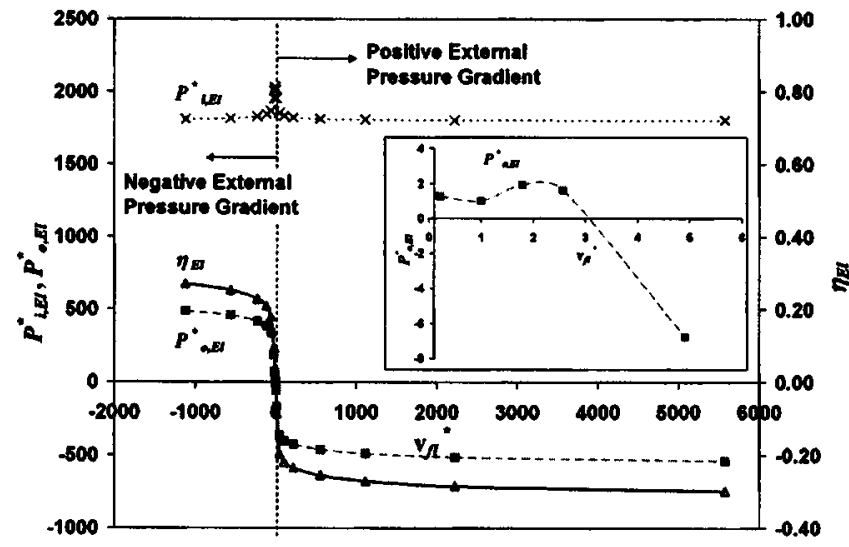

(b)

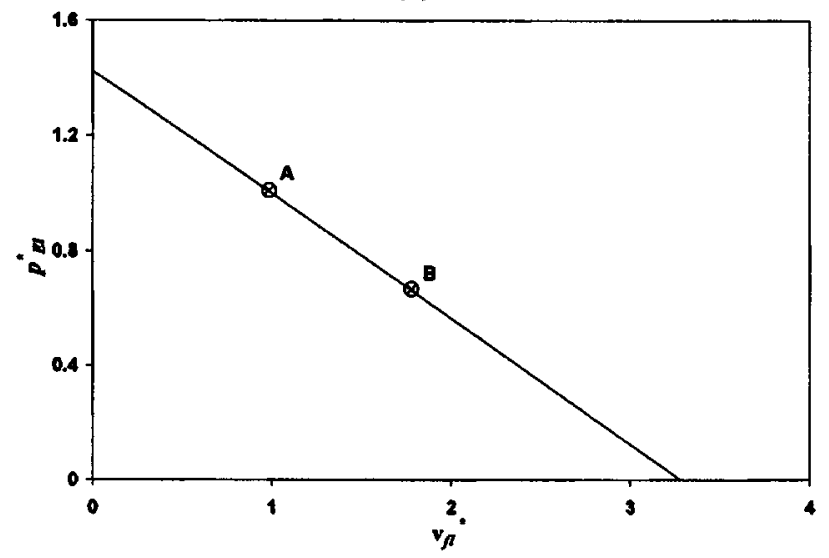

(c)

Fig. 8 Variation of nondimensional total input power, total output power, and total efficiency; (b) nondimensional electrical input power, output power due to EHD, and efficiency due to EHD; and (c) pump curve, i.e., nondimensional pressure head generated by the pump with nondimensional bulk fluid velocity due to combined action of the attraction-type induction EHD pump and varying external pressure gradient

the pumps considered, an attraction-type EHD pump, are plotted in Fig. 8. Figure $8(a)$ shows that $P_{i, \text { tot }}^{*}, P_{o \text {,tot }}^{*}$, and $\eta_{\mathrm{tot}}$ vary with $v_{\mathrm{fl}}^{*}$ in a manner similar to the behavior of the previously discussed pumps. The variation of $P_{i, \mathrm{E} \ell}^{*}, P_{o, \mathrm{E} \ell}^{*}$, and $\eta_{\mathrm{E} \ell}$ with $v_{\mathrm{fl}}^{*}$ is shown in Fig. $8(b)$; the inset is a magnified view of the behavior at low velocities. The variation of $P_{i, \mathrm{E} \ell}^{*}$ with $v_{\mathrm{fl}}^{*}$ is interesting, with its largest value being very close to $v_{\mathrm{fl}}^{*}=1$, decreasing at both larger and smaller values of $v_{\mathrm{fl}}^{*}$. At very large and very small values of $v_{\mathrm{fl}}^{*}$, little variation in $P_{i, \mathrm{E} \ell}^{*}$ is noticed.

The drop in $P_{i, \mathrm{E} \ell}^{*}$ as $v_{\mathrm{fl}}^{*}$ increases can be explained as follows. The attraction-type induction EHD pump is a synchronous pump [13]. EHD action tries to move the fluid at the same velocity as the potential wave. When the fluid velocity is smaller than the velocity of the potential wave, the pump operates in a "pumping" mode, where it tries to increase the velocity of the fluid, which is limited by the electrical power available and the viscous forces. If the fluid velocity is higher than the wave velocity, the pump goes into a "braking" mode, where it tries to slow down the fluid velocity to the synchronous speed. Here, the pump is limited only by the electrical power available. Hence the largest fluid velocity achievable in an attraction-type induction EHD pump, in the absence of external pressure gradients, is the wave velocity itself. For the present case, the synchronous speed is approximately $v_{\mathrm{fl}}^{*}$ $=3.3$. The output power from $\operatorname{EHD}\left(P_{o, \mathrm{E} \ell}^{*}\right)$ becomes negative at around this value, as can be seen more clearly from the inset. Similarly, if the fluid velocity decreases below the synchronous speed, $P_{o, \mathrm{E} \ell}^{*}$ increases rapidly. The negligible variation in $P_{i, \mathrm{E} \ell}^{*}$ at large absolute values of $v_{\mathrm{fl}}^{*}$ is due to the insignificant effect of variation in charge convection as the net charge in the fluid is zero.

The pump curve for the present pump is shown in Fig. 8(c). The pump generates a net positive pressure gradient along with flow in the positive direction only for $v_{\mathrm{fl}}^{*}<3.3$. The maximum fluid output power in this case (point $B$ ) is approximately $20 \%$ greater than the power in the absence of an external pressure gradient (point A). It is interesting to note that the pump ceases to generate a net positive pressure gradient at $v_{\mathrm{fl}}^{*}$ close to 3.3 , as it goes into the braking mode beyond this point.

\section{Conclusions}

The efficiency of EHD pumping depends strongly on the bulk fluid velocity. For flow due to a constant, stationary potential gradient, as well as for flow due to repulsion-type induction EHD, the efficiency of EHD pumping increases monotonically with an increase in the bulk fluid velocity. Moreover, the total fluid power output from the combined action of EHD and an externally imposed pressure gradient is larger than the sum of fluid power outputs from their action independent of each other.

The variation of efficiency of an ion-drag EHD pump driven by a traveling potential wave and a transient source of charge density with the bulk fluid velocity depends on the ratio of bulk fluid velocity to the wave velocity. The efficiency of the pump increases with increasing bulk fluid velocity for small values of this ratio and it decreases for large values of this ratio. For attractiontype induction EHD pumps, with flow in the forward direction, the efficiency of EHD action is highest when the fluid velocity is equal to the wave velocity. For fluid velocities in the forward direction larger than the wave velocity, the pump acts to retard the flow.

Results for the input electrical power, output fluid power, and efficiency of EHD action are provided as a function of bulk fluid velocity for the five different EHD pumps considered; graphical pump curves for all the pumps are also developed.

\section{Acknowledgment}

Support in the form of a fellowship for the first author, provided by the Purdue Research Foundation, is gratefully acknowledged.

\section{Nomenclature}

$$
\begin{aligned}
A & =\text { cross-sectional area }\left(\mathrm{m}^{2}\right) \\
D & =\text { charge diffusion coefficient }\left(\mathrm{m}^{2} / \mathrm{s}\right) \\
E & =\text { electric field }(\mathrm{V} / \mathrm{m}) \\
E s & =\text { electric source number (dimensionless) }
\end{aligned}
$$




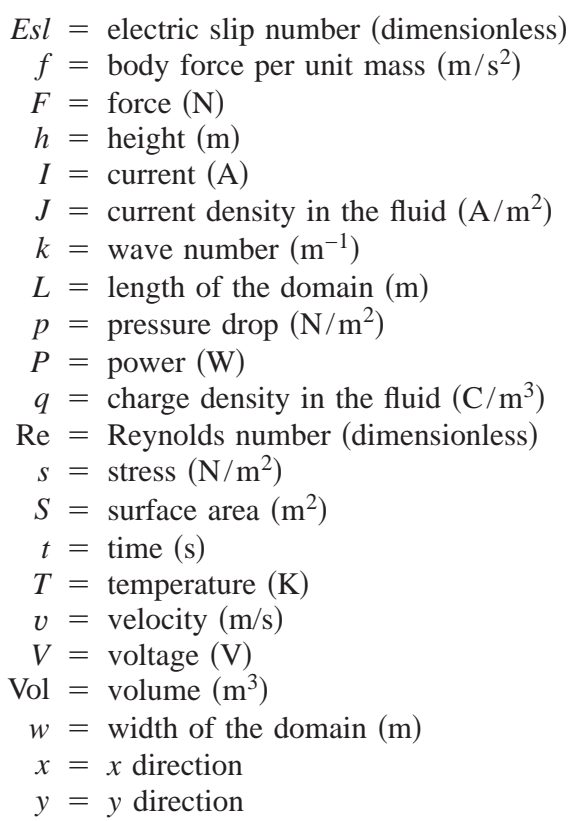

Subscripts and superscripts

$*=$ nondimensional

app $=$ applied

$c=$ collector

$d=$ drift

$e=$ emitter

$E=$ electric only (without external pressure gradient)

$\mathrm{EHD}=$ with EHD

$\mathrm{E} \ell=$ net electric

$f l=$ fluid

$i=$ input

ion $=$ ion

$m=$ mechanical

$\max =$ maximum value (value at the upstream electrode)

No EHD = without EHD

$o=$ output

tot $=$ total

wa $=$ weighted average

$x=x$ coordinate

$y=y$ coordinate

$z=z$ coordinate

\section{Greek symbols}

$\alpha=$ loss coefficient (dimensionless)

$\delta_{i j}=$ Kronecker delta vector (dimensionless)

$\varepsilon=$ permittivity of the fluid $(\mathrm{F} / \mathrm{m})$

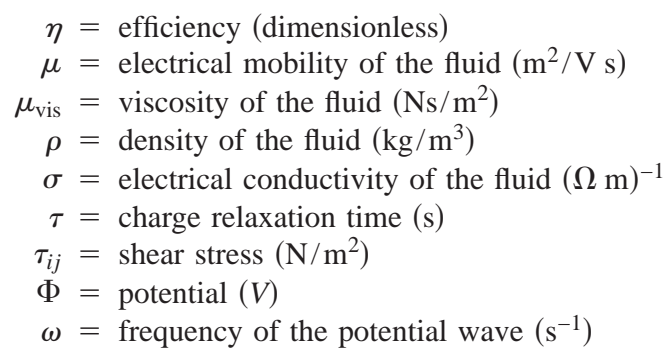

References

[1] Stuetzer, O., 1959, “Ion Drag Pressure Generation,” J. Appl. Phys., 30, pp. 984-994.

[2] Robinson, M., 1961, "Movement of Air in the Electric Wind of the Corona Discharge," J. Am. Inst. Electr. Eng., 80, pp. 143-150.

[3] Pickard, W., 1963, "Ion Drag Pumping. II. Experiment," J. Appl. Phys., 34 pp. 251-258.

[4] Melcher, J. R., 1966, "Traveling-Wave Induced Electroconvection," Phys. Fluids, 10, pp. 1548-1555.

[5] Gerdt, R. E., Crowley, J. M., and Chato, J. C., 1978, "Electrohydrodynamic Pumping of Cable Oil," J. Electrost., 5, pp. 477-488.

[6] Richter, A., Plettner, A., Hofmann, K. A., and Sandmaier, H., 1991, “A Micromachined Electrohydrodynamic (EHD) Pump," Sens. Actuators, A, 29, pp. $159-168$.

[7] Ahn, S.-H., and Kim, Y.-K., 1998, "Fabrication and Experiment of a Planar Micro Ion Drag Pump," Sens. Actuators, A, 70, pp. 1-5.

[8] Bart, S. F., Tavrow, L. S., Mehregany, M., and Lang, J. H., 1990, "Microfabricated Electrohydrodynamic Pumps," Sens. Actuators, A, 21-23, pp. 193 197.

[9] Fuhr, G., Hagedorn, R., Muller, T., Benecke, W., and Wagner, B., 1992, "Microfabricated Electrohydrodynamic (EHD) Pumps for Liquids of Higher Conductivity," J. Microelectromech. Syst., 1, pp. 141-146.

[10] Manz, A., Effenhauser, C. S., Burggraf, N., Harrison, D. J., Seiler, K., and Fluri, K., 1994, "Electroosmotic Pumping and Electrophoretic Separations for Miniaturized Chemical Analysis Systems,” J. Micromech. Microeng., 4, pp. 257-265.

[11] Zeng, S., Chen, C. H., Mikkelsen, Jr., J. C., and Santiago, J. C., 2001, "Fabrication and Characterization of Electroosmotic Micropumps," Sens. Actuators B, 79, pp. 107-114.

[12] Bondar, H., and Bastien, F., 1986, "Effect of Neutral Fluid Velocity on Direct Conversion from Electrical to Fluid Kinetic Energy in an Electro-FluidDynamics (EFD) Device,” J. Phys. D, 19, pp. 1657-1663.

[13] Crowley, J. M., 1980, "The Efficiency of Electrohydrodynamic Pumps in the Attraction Mode," J. Electrost., 8, pp. 171-181.

[14] Crowley, J. M., Wright, G. S., and Chato, J. C., 1990, "Selecting a Working Fluid to Increase the Efficiency and Flow Rate of an EHD Pump," IEEE Trans. Ind. Appl., 26, pp. 42-49.

[15] Seyed-Yagoobi, J., Bryan, J., and Castaneda, J., 1995, "Theoretical Analysis of Ion-Drag Pumping," IEEE Trans. Ind. Appl., 31, pp. 469-476.

[16] Singhal, V., and Garimella, S. V., 2004, "A Novel Micropump for Electronics Cooling," International Mechanical Engineering Congress and Exposition, IMECE2004-61147, Anaheim, CA, 1-12.

[17] Singhal, V., and Garimella, S. V., 2004, "A Novel Valveless Micropump with Electrohydrodynamic Enhancement for High Heat Flux Cooling,” IEEE Trans. Adv. Packag. (accepted for publication, in press).

[18] FIDAP 8.7.2, FIDAP User's Manual, Fluent, Inc., Lebanon, NH, 2003.

[19] Melcher, J. R., and Firebaugh, M. S., 1967, "Traveling-Wave Bulk Electroconvection Induced Across a Temperature Gradient,” Phys. Fluids, 10, pp. 1178 1185 